\title{
Surgical Management Strategy for Breast Cancer Patients During the COVID-19 Outbreak
}

This article was published in the following Dove Press journal:

Cancer Management and Research

\author{
Yong Liu (iD)' \\ Jiaming Wang' \\ Yi Tang' \\ Rong Su' \\ Qianwen Yang ${ }^{2}$ \\ 'The Third Affiliated Hospital of \\ Guangzhou Medical University, \\ Guangzhou, People's Republic of China, \\ 5I0I50; ${ }^{2}$ The University of Melbourne, \\ Melbourne, Australia, 3052
}

\begin{abstract}
In December 2019, a new coronavirus pneumonia began to break out globally. COVID-19 pandemic challenges the health systems worldwide and influences the treatments for other diseases. The incidence rate of breast cancer ranks first among all malignant tumors among women. During the pandemic, medical workers should strictly monitor the condition of patients and strengthen the management and prevention measures to make sure patients can be operated safely. This article will discuss the arrangements and management of surgical treatment for patients with breast cancer.
\end{abstract}

Keywords: COVID-19, pneumonia, breast cancer, surgery

\section{Introduction}

In December 2019, a cluster of pneumonia cases with unknown causes was reported globally. ${ }^{1}$ Then Chinese scientists identified a novel coronavirus by gene sequencing and isolated the virus in a very short time. ${ }^{2,3}$ The coronavirus, which was named as Novel Coronavirus 2019 (2019-nCov) by WHO is highly infectious. ${ }^{4,5}$ On February 11, WHO announced "COVID-19" as the name of the disease caused by 2019-nCov. Globally, by April 5, 1,133,758 confirmed cases and 62,784 death have been reported. ${ }^{6}$ Many countries have taken active measures to control the outbreak. Some cities were "locked down" to cut off the transmission routes, however, the measures also affected people's regular life and challenged the health system.

Breast cancer is the most common malignant tumor among women. According to Global cancer statistic 2018, an estimated 2.1 million new cases of breast cancer were expected to be diagnosed in $2019 .^{7}$ For early-stage breast cancer, surgery is the primary treatment. Mateo et al's study indicated that delaying surgical treatment of patients with operable breast cancer would have a negative impact on the prognosis, with 1.104 times increased risk of death for each month of delay. ${ }^{8}$ Patients with breast cancer may not be able to receive treatments on time due to the outbreak. The approach to carry out surgical treatment for patients in need under strict protection is a challenge faced by the medical worker in Breast Surgery. This article is largely based on the recommendations of the Chinese National Health Commission, and the practices of our hospital and departments on the surgical management strategy for breast cancer patients under the new type of coronary pneumonia. Our department treated more than 400 patients after the outbreak, and none of them was infected with COVID-19 under our management strategy.
Correspondence: Yong Liu

The Third Affiliated Hospital of

Guangzhou Medical University,

Guangzhou 510150, People's Republic of

China

Email dr13809207569@163.com 


\section{Relationship Between COVID-I9 and Patients with Breast Cancer}

A study conducted in China found $18(1 \%)$ of the total 1590 COVID-19 patients confirmed by January 21 had a history of cancer, while in China, the incidence of cancer among the whole population is $0.29 \%$ (285.83 per 100000 person-years). ${ }^{9}$ It seemed the incidence of COVID-19 among people with cancer is higher than the average. ${ }^{9}$ Besides, patients with cancer had a higher risk of adverse outcomes, including being transferred to ICU and death compared with patients without cancer (39\% VS 8\%, $\mathrm{P}=0.0003) .{ }^{9}$ However, Yang et al reviewed the medical histories of the 18 patients in the above study and found more than half of them had recovered from the previous cancer treatments and were clinically cured. ${ }^{10}$ Furthermore, the small sample size and considerable heterogeneity suggested the result might be inappropriate to be generalized to the entire population with cancer. ${ }^{10}$

Studies showed among COVID-19 patients, severe inflammatory response and cytokine related lung damage could induce severe pneumonia events. ${ }^{11}$ Cancer is often associated with low immunity. The overexpression of immunosuppressive cytokines inhibits inflammation, which contradicts the idea that patients with cancer are prone to develop severe COVID-19 events. However, CCDC did an epidemiology descriptive studies that involved the 72,314 cases (including confirmed, suspected and clinically diagnosed cases) reported by January 11 and found among patients with underlying diseases, 107 $(0.5 \%)$ of them had cancer, and six of the 107 died, with a crude mortality rate of $5.6 \%$, higher than the average mortality $(2.3 \%) .^{12}$

Though the evidence remains insufficient, patients with breast cancer are expected to have a higher risk of COVID-19 since (1) their immune system are impacted by surgery, radiotherapy, chemotherapy, and immunotherapy; (2) breast cancer happens more among the elder people and old ages link to poorer health conditions; (3) patients need to go to the hospital for treatments, which increase their risk of exposure. ${ }^{13}$ Besides, once patients with cancer, including breast cancer patients are infected by 2019 -nCov, not only the treatment for cancer must be delayed, they are also more likely to develop severe pneumonia (ie, admission to the intensive care unit, invasive ventilation, or death seen in 7 of 18 patients [39\%] with cancer vs 124 of
1572 patients [8\%] without cancer; $\mathrm{p}=0.0003) .{ }^{9}$ Therefore, patients with breast cancer have a high risk in the COVID-19 outbreak.

\section{Screening Strategies and Hospitalization Criteria for Breast Cancer Under COVID-19 Outbreak}

During the outbreak, it is necessary to adjust the hospitalization criteria and the rules of protection in the hospitals. Researches have revealed the nosocomial infection ratio of COVID-19 was $43 \% .{ }^{14}$ The exclusion criteria of hospitalization include fever (body temperature $\geq 37.3^{\circ} \mathrm{C}$ ), cough, coughing up sputum or phlegm and other respiratory symptoms. If patients showed the above symptoms, they must go to the fever clinics (a department set up during the period of acute infectious disease prevention and control, targeting to treat fever patients and screen suspected cases. The initial symptoms of COVID-19, including fever and respiratory symptoms are nonspecific. The major task of fever clinics is identifying COVID-19 patients from other patients who merely present COVID-19-like symptoms ${ }^{15}$ ). Moreover, patients who have been to the major epidemic area or have been in close contact with a confirmed or suspected COVID-19 case are required to be quarantined at home for 14 days, during which time the patient cannot be hospitalized for surgery. Before being hospitalized, patients must have blood routine tests(analyze the plasma levels of leukocyte, platelets, C-reactive protein (CRP), neutrophil, D-dimer, procalcitonin and so on ${ }^{16,17}$ ) and realtime reverse transcription polymerase chain reaction (RTPCR) of viral nucleic acid. However, recent studies addressed the importance of chest computed tomography (CT) examination in COVID-19 patients with false negative RT-PCR results, ${ }^{18,19}$ and reported the CT sensitivity as $98 \%{ }^{20}$ Additionally, according to the official diagnosis and treatment protocol (6th edition) declared by the National Health Commission of China, ${ }^{21}$ CT examination is of great significance not only in diagnosing COVID-19 but also in monitoring disease progression and evaluating therapeutic efficacy. Therefore, for conditional hospitals and medical clinics, admitted patients should also have routine chest CT examination, But for asymptomatic patients who have not suspicious contacts and who have a negative coronavirus nucleic acid test, $\mathrm{CT}$ examination is not necessary ${ }^{22}$. If the patients do not meet any exclusive criteria and all the tests results show negative, they can be hospitalized after appointments. 
In addition, we suggest the hospitalization criteria for patients who need to be operated in a certain time limit as follows: (1) early-stage patients that confirmed by biopsy and other pathological examination; (2) people that are highly suspected of breast cancer according to the clinical and radiographic examination but not confirmed by biopsy; (3) breast cancer patients who need surgery to complete neoadjuvant chemotherapy. Although the proposal is different from the previous consensus in the literature and recommendations, ${ }^{23}$ it is the most reasonable and practical measures in view of the present conditions of our community and hospitals.

\section{Perioperative Management}

During the outbreak of COVID-19, all medical institutions are required to establish a health status reporting system. ${ }^{24}$ All health workforce in the hospital, including medical practitioners, security guards, cleaners, and caregivers, etc. need to monitor and report their health status every day. In terms of the infection control procedures, we shall refer to the Technical Guidelines for the Prevention and Control of New Coronavirus Infections in Medical Institutions (First Edition), ${ }^{25}$ preventing the nosocomial infection from the dimensions of system, organizing, medical training, etc. Under the guidance, no nosocomial infection occurred in the Vulcan Hill Hospital, where has admitted 2404 patients by 10 March $2020 .^{26}$ To avoid the overuse of scarce medical resource, the author believes that under the premise of strict prevention measures in the hospitals, the health examination for medical practitioners only requires a nasopharyngeal swab test before resumption of work and a daily monitoring of body temperature.

The patients shall be accompanied by only one person, whose 2019-nCov screening result is negative. Although the presence of caregivers in the hospital could be a vehicle of infection, their presence is of great help to patients both physically and psychologically. ${ }^{27}$ During the hospitalization, the patients and accompanying person shall wear masks and take daily body temperature measurement. ${ }^{28}$ If the body temperature higher than $37.3^{\circ} \mathrm{C}$, enhanced protection should be applied in the ward. For example, we can transit patients with fever into a separate ward or a buffer ward till excluding the positive results. ${ }^{24}$ Medical workers should analyse the reason for the fever and refer the individuals to the fever clinic for further treatment if necessary. Comprehensive preoperative preparation and preoperative discussion are needed to ensure a safe surgery. The principle of aseptic operation must be strictly observed during the surgery. The medical workers involved in the operation can wear goggles and double gloves. Patients should wear a mask before endotracheal intubation and after extubation. ${ }^{29}$ Medical workers must adhere to the daily body temperature measurements towards patients and strengthen the management of respiratory tract infection. If the patients have a fever, repeat the blood routine first to identify the surgical infection and bleeding. Do the nucleic acid test and chest CT again to rule out COVID-19 if necessary. After discharge, all items used by the patients, such as bed and clothes should be disinfected to avoid crossinfection. ${ }^{30}$

\section{Postoperative Treatment and Postoperative Follow-Up}

Except under special circumstances, patients should avoid going to the hospital to decrease the risk of infection during the journey and in the hospital. Postoperative follow-ups that instruct patients to exercise properly after surgery can be done via telephone and the internet.

Patients who need adjuvant chemotherapy and targeted therapy after the surgery may need to adjust the time of treatment due to the COVID-19 outbreak. The best time for adjuvant therapy for breast cancer is 3-6 weeks after surgery, as recommended by the European society of medical oncology clinical practice guidelines. ${ }^{31} \mathrm{Li}$ et al included 303 patients with stage I-III breast cancer in their study and found that it was beneficial to prolong the DFS rate of breast cancer when started the postoperative adjuvant chemotherapy within 28 days after the surgery, ${ }^{32}$ suggesting that the optimal time of postoperative adjuvant chemotherapy is $22-28$ days after the surgery. However, during the pandemic period, the starting time of chemotherapy can be extended appropriately according to the pathological characteristics of patients. A study indicated that patients with breast cancer of high risk of recurrence, such as those showed high Ki-67 and HER-2 or triple-negative breast cancer should receive chemotherapy within eight weeks, while patients with low risk of recurrence can receive the chemotherapy within 90 days. ${ }^{33}$ Therefore, we believe that the principle of chemotherapy for patients during the epidemic should be similar to that in the past, and treatment can be carried out as planned under the premise of objective conditions permitting and ensuring protective safety. If the conditions 
do not permit, strengthen the contact with the case manager and make an appointment for treatment.

Radiotherapy is also an important part of breast cancer treatment. Patients who require radiotherapy after the total mastectomy should be back to the hospital for radiotherapy within 2-4 weeks after the completion of the last chemotherapy. ${ }^{34}$ For patients receiving adjuvant chemotherapy, postoperative radiotherapy is recommended to be completed within 8 weeks. ${ }^{34}$ Therefore, the interval of radiotherapy can be extended to $4-8$ weeks. Regarding the endocrine treatment of breast cancer after surgery, the Guidelines and Guidelines for the Diagnosis and Treatment of Breast Cancer of the China Anti-Cancer Association recommends that it be carried out after chemotherapy. ${ }^{35}$ However, during the outbreak, the patient was in a state of home isolation. Therefore, China's health insurance department issued the corresponding policy for cancer patients, so that patients with endocrine therapy after cancer surgery can prescribe a maximum dose of 90 days in the outpatient clinic. ${ }^{36}$ Therefore, during the epidemic, endocrine therapy for patients should not be delayed or stagnated.

\section{Mental Health Intervention}

COVID-19 is a major public health emergency and is highly contagious. People's psychological pressure tends to increase in such a circumstance. The National Health Committee issued Guidelines for Emergency Psychological Crisis Intervention in COVID-19 Outbreak on January $27 .{ }^{37}$

Breast cancer patients are more vulnerable to developing anxiety, depression and other negative emotions compared with healthy individuals. The COVID-19 pandemic aggravated the vulnerability. It is common that patients concern about being infected by the coronavirus and how to access to health services of oncology during the pandemic. $^{38}$ Health workers and family members should pay attention to patients' mental health and help them stay positive. Patients should be encouraged to seek psychological help when necessary.

Medical workers are on the front-line of the pandemic, not only undergoing intensive work but also facing great psychological pressure. Medical workers can use helpful coping strategies such as staying connected with family and friends, taking sufficient rest, and eating healthy food. ${ }^{39}$ Hospitals should provide psychological counselling and related training to medical workers, helping them do their duty and stay in a good mental state. Some telemedicine platforms in China also offer free psychological counselling for people in need. ${ }^{40}$

Nowadays, COVID-19 is sweeping the world. All health workers and patients must face it together, take strict protections, and conquer the virus soon. For breast cancer patients who need surgery, individual management and scientific self-preventions are required. With appropriate management, we can control the outbreak and fight against cancer at the same time.

\section{Author Contributions}

All authors contributed to data analysis, drafting or revising the article, gave final approval of the version to be published, and agree to be accountable for all aspects of the work.

\section{Funding}

The authors received no financial support for the research, authorship, or publication of this article.

\section{Disclosure}

The authors declare that they have no competing interests.

\section{References}

1. China CDC. 2019 Novel Coronavirus (2019-Ncov). Wuhan, China: Centres for Disease Control and Prevention; 2020. Available from: https://www.cdc.gov/coronavirus/2019-ncov/index.html.

2. Wu F, Zhao S, Yu B, et al. A new coronavirus associated with human respiratory disease in China. Nature. 2020;579(7798):265-269. doi:10.1038/s41586-020-2008-3

3. Zhu N, Zhang D, Wang W, et al. A novel coronavirus from patients with pneumonia in China, 2019. N Engl J Med. 2020;382(8):727-733. doi:10.1056/NEJMoa2001017

4. Hui DS, Azhar E, Madani TA, et al. The continuing 2019-nCoV epidemic threat of novel coronaviruses to global health - The latest 2019 novel coronavirus outbreak in Wuhan, China. Int $J$ Infect Dis. 2020;91:264-266. doi:10.1016/j.ijid.2020.01.009

5. The World Health Organization. Report of the WHO-China Joint Mission on Coronavirus Disease 2019 (COVID-19). Geneva: The World Health Organization; 2020. Available from: https://www.who. int/docs/default-source/coronaviruse/who-china-joint-mission-oncovid-19-final-report.pdf.

6. The World Health Organization. Coronavirus Disease 2019 (COVID19) Situation Report-76. Geneva: The World Health Organization; 2020. Available from: https://www.who.int/docs/default-source/corona viruse/situation-reports/20200405-sitrep-76-covid-19.pdf?sfvrsn= 6ecf0977 2.

7. Bray F, Ferlay J, Soerjomataram I, et al. Global cancer statistics 2018: GLOBOCAN estimates of incidence and mortality worldwide for 36 cancers in 185 countries. CA Cancer J Clin. 2018;68(6):394-424. doi:10.3322/caac. 21492

8. Mateo AM, Mazor AM, Obeid E, et al. Time to Surgery and the Impact of Delay in the Non-Neoadjuvant Setting on Triple-Negative Breast Cancers and Other Phenotypes. Ann Surg Oncol. 2019. doi:10.1245/s10434-019-08050-y

9. Liang W, Guan W, Chen R, et al. patients in SARS-CoV-2 infection: a nationwide analysis in China. Lancet Oncol. 2020;21(3):335-337. doi:10.1016/S1470-2045(20)30096-6 
10. Wang H, Zhang L. Risk of COVID-19 for patients with cancer. Lancet Oncol. 2020;21(4):e181. doi:10.1016/S1470-2045(20)30149-2

11. Xu Z, Shi L, Wang Y, et al. Pathological findings of COVID-19 associated with acute respiratory distress syndrome. Lancet Respir Med. 2020;8(4):420-422. doi:10.1016/S2213-2600(20)30076-X

12. Novel Coronavirus Pneumonia Emergency Response Epidemiology Team. The epidemiological characteristics of an outbreak of 2019 novel coronavirus diseases (COVID-19) in China. Zhonghua Liu Xing Bing Xue Za Zhi. 2020;41(2):145-151. doi:10.3760/cma.j. issn.0254-6450.2020.02.003

13. Yu J, Ouyang W, Chua MLK, Xie C. SARS-CoV-2 Transmission in Patients With Cancer at a Tertiary Care Hospital in Wuhan, China [published online ahead of print, 2020 Mar 25]. JAMA Oncol. 2020; e200980. doi:10.1001/jamaoncol.2020.0980

14. Wang $\mathrm{D}, \mathrm{Hu} \mathrm{B}, \mathrm{Hu} \mathrm{C}$, et al. Clinical Characteristics of 138 Hospitalized Patients With 2019 Novel Coronavirus-Infected Pneumonia in Wuhan, China. JAMA. 2020;323(11):1061-1069. doi:10.1001/jama.2020.1585

15. Li Q, Ding X, Xia G, et al. Eosinopenia and elevated C-reactive protein facilitate triage of COVID-19 patients in fever clinic: a retrospective case-control study [published online ahead of print, 2020 May 3]. EClinicalMedicine. 2020:100375. doi:10.1016/j. eclinm.2020.100375.

16. Terpos E, Ntanasis-Stathopoulos I, Elalamy I, et al. Hematological findings and complications of COVID-19. Am J Hematol. 2020:1-14. doi:10.1002/ajh.25829.

17. Soraya, Gita Vita and Ulhaq, Zulvikar Syambani, Crucial Laboratory. Parameters in COVID-19 Diagnosis and Prognosis: an Updated Meta-Analysis. 2020. doi:10.2139/ssrn.3576912

18. Xie X, Zhong Z, Zhao W, Zheng C, Wang F, Liu J. Chest CT for typical 2019-nCoV pneumonia: relationship to negative RT-PCR testing. Radiology. 2020;200343. doi:10.1148/radiol.2020200343

19. Huang P, Liu T, Huang L, et al. Use of Chest CT in Combination with Negative RT-PCR Assay for the 2019 Novel Coronavirus but High Clinical Suspicion. Radiology. 2020;295(1):22-23. doi:10.1148/ radiol.2020200330

20. Fang Y, Zhang H, Xie J, et al. Sensitivity of chest CT for COVID-19: comparison to RT-PCR. Radiology. 2020:200432. doi:10.1148/ radiol.2020200432

21. National Health Commission of the People's Republic of China. The Diagnostic and Treatment Protocol of COVID-19. China; 2020. Available from: http://www.gov.cn/zhengce/zhengceku/2020-02/19/ content 5480948.htm. Accessed 3 Mar 2020.

22. Gao Z, Xu Y, Sun C, et al. A Systematic Review of Asymptomatic Infections with COVID-19 [published online ahead of print, 2020 May 15]. J Microbiol Immunol Infect. 2020. doi:10.1016/j. jmii.2020.05.001

23. Motlagh A, Yamrali M, Azghandi S, et al. COVID19 Prevention \& Care; A Cancer Specific Guideline. Arch Iran Med. 2020;23(4):255264. doi:10.34172/aim.2020.07

24. Announcement of strengthening the infection control in medical institutions during the COVID-19 outbreak by the National Health Commission; 2020. Available from: http://www.gov.cn/zhengce/ zhengceku/2020-03/13/content 5491044.htm

25. National Health Commission. Technical Guide for novel coronavirus infection Prevention and Control in Medical institutions (first Edition). Chin J Infect Control. 2020;19(02):189-191.
26. Hongmei F, Khabsa J, Solo K, et al. Nosocomial infection control and prevention in COVID-19 specialist Hospital- take Huoshenshan Hospital as an example. Mil Med. 2020:1-4.

27. Curigliano G, Cardoso MJ, Poortmans P, et al. Recommendations for triage, prioritization and treatment of breast cancer patients during the COVID-19 pandemic [published online ahead of print, 2020 Apr 16]. Breast. 2020;52:8-16. doi:10.1016/j.breast.2020.04.006

28. Zhen L, Lin T, Zhao ML, et al. Management strategy for the resumption of regular diagnosis and treatment in gastrointestinal surgery department during the outbreak of coronavirus disease 2019 (COVID-19). Zhonghua Wei Chang Wai Ke Za Zhi. 2020;23 (4):321-326. doi:10.3760/cma.j.issn.1671-0274.2020-0316-00146

29. Zimmermann M, Nkenke E. Approaches to the management of patients in oral and maxillofacial surgery during COVID-19 pandemic. J Craniomaxillofac Surg. 2020;48(5):521-526. doi:10.10 16/j.jcms.2020.03.011

30. De Simone B, Chouillard E, Di Saverio S, et al. Emergency surgery during the COVID-19 pandemic: what you need to know for practice. Ann $R$ Coll Surg Engl. 2020;102(5):323-332. doi:10.1308/rcsann. 2020.0097

31. Cardoso F, Kyriakides S, Ohno S, et al. breast cancer: ESMO Clinical Practice Guidelines for diagnosis, treatment and follow-up. Ann Oncol. 2019;30(8):1194-1220. doi:10.1093/annonc/mdz173

32. Melchior NM, Sachs DB, Gauvin G, et al. Treatment times in breast cancer patients receiving neoadjuvant vs adjuvant chemotherapy: is efficiency a benefit of preoperative chemotherapy? Cancer Med. 2020;9(8):2742-2751. doi:10.1002/cam4.2912

33. Yu KD, Fan L, Qiu LX, et al. Influence of delayed initiation of adjuvant chemotherapy on breast cancer survival is subtype-dependent. Oncotarget. 2017;8(28):46549-46556. doi:10.18 632/oncotarget. 10551

34. Health Commission of PRC N; National Health Commission Of The People's Republic Of China. Chinese guidelines for diagnosis and treatment of breast cancer 2018 (English version). Chin J Cancer Res. 2019;31(2):259-277. doi:10.21147/j.issn.1000-9604.2019.02.02

35. Of the People, N.H.C. Guidelines and norms for breast cancer diagnosis and treatment of China Anti-Cancer Association (2019 edition). Chin J Cancer. 2019;29(08):609-680.

36. Tai XU, et al. Talking about the management of breast cancer patients during the epidemic period of COVID-19. Chin J Breast Dis. $2020 ; 1-3$.

37. The National Health Committee. Guiding Principles of Emergency Psychological Crisis Intervention in Novel Coronavirus Pneumonia. Beijing: State Council of the People's Republic of China; 2020.

38. Al-Shamsi HO, Alhazzani W, Alhuraiji A, et al. A Practical Approach to the Management of Cancer Patients During the Novel Coronavirus Disease 2019 (COVID-19) Pandemic: an International Collaborative Group [published online ahead of print, 2020 Apr 3]. Oncologist. 2020. doi:10.1634/theoncologist.2020-0213

39. The World Health Organization. Mental health and psychosocial considerations during the COVID-19 outbreak (2020.3.18); 2020. Available from: https://apps.who.int/iris/bitstream/handle/10665/ 331490/WHO-2019-nCoV-MentalHealth-2020.1-eng.pdf.

40. Chen. G, More users choose telemedicine during the COVID-19 outbreak (2020.3.14); 2020. Available from: http://www.xinhuanet. com/info/2020-03/14/c 138876278.htm. 


\section{Publish your work in this journal}

Cancer Management and Research is an international, peer-reviewed The manuscript management system is completely online and includes open access journal focusing on cancer research and the optimal use of a very quick and fair peer-review system, which is all easy to use. preventative and integrated treatment interventions to achieve improved Visit http://www.dovepress.com/testimonials.php to read real quotes outcomes, enhanced survival and quality of life for the cancer patient.

from published authors. 